\title{
An Interaction Design Analysis of Mood Trackers
}

\author{
Rita Branco ${ }^{1}$, Marco Neves ${ }^{2}$, Paulo Noriega ${ }^{2}$, and Mafalda Casais ${ }^{2}$ \\ ${ }^{1}$ Lisbon School of Architecture, University of Lisbon, Rua Sá Nogueira, Pólo \\ Universitário, Alto da Ajuda, 1349-055 Lisbon, Portugal \\ ${ }^{2}$ CIAUD - Research Center for Architecture, Urbanism and Design, Lisbon School of \\ Architecture, University of Lisbon, Rua Sá Nogueira, Pólo Universitário, Alto da \\ Ajuda, 1349-055 Lisbon, Portugal \\ branco1@campus.ul.pt \\ mneves@fa.ulisboa.pt \\ pnoriega@fa.ulisboa.pt \\ mafaldacasais.ciaud@fa.ulisboa.pt
}

\begin{abstract}
Mood trackers are very prevalent nowadays; however, most of them are designed for the general public, without much concern for mental health issues or targeting people in therapy. The aim of this paper is to report an introductory analysis of what mood tracker applications in the present look like, pointing out strong points and some negative sides. Also, it aims to define what can be useful for future improvement by detecting patterns within these applications. Our research methodology comprises an analysis of a selection of applications found in the Google App Store and Apple Store, through a series of different criteria taken from the relevant literature. Our results suggest that the incorporation of guidelines, co-design methods with mental health specialists, and new interaction styles used, could potentially change the mood tracker market for the better. Further studies are necessary to demonstrate the impact of images in improving mood trackers and their use in the context of therapy.
\end{abstract}

Keywords: Mood Trackers, Interaction Design, Mobile Mental Health, Mental

Health, Mobile Applications

\section{$1 \quad$ Introduction}

Mental health mobile applications are, nowadays, very common [1]; and the fact that they have become more common has a positive impact on mental health in general because it brings a "reduction of stigma associated with seeking mental health treatment" [2]. However, most are not supported by professionals in the field, and more rigorous research and design guidelines for the functionalities and interfaces of mental health mobile applications 
available for smartphones are needed [3]. A lot of these applications have their approach centered around the use of mood trackers, which are defined by Caldeira [3] as "an approach to help healthy individuals stay in healthy emotional states, and assist individuals with mental diseases" by registering moods and emotions in a timeframe. Mood trackers help people get a better overview of their emotional state, by learning about their mood patterns and reflecting on them, which helps manage emotional distress.

Mood differs from emotions - which are short-lived reactions to stimuli -, and can be defined as "a mild, diffuse, pervasive feeling state that is experienced as pleasant or unpleasant, and which has a broad influence on perceptions, motivation, and behaviour" [4]. Mood is interesting to study and to record in the context of mental health because it "has a direct influence on people's subjective well-being," i.e., their happiness [4]. An example of a mood tracker used by soldiers is a mobile application that enables users to rate their moods, to self-monitor across time, and to report their emotional experiences to health providers, with the purpose of tracking and preventing symptoms associated with deployment-related behavioral health issues, including post traumatic stress disorder, head injury, stress, depression, anxiety, and general well-being [5].

Currently the market is overflowing with mood tracker applications; one quick search using the keywords "mental health" or "mood tracker" in any application store will lead us to a numerous amount of options to choose from. However, most of these are not specifically designed for individuals that suffer from mental illness or people who currently go to therapy, rather focusing on the general population [3]. As a consequence, the current widespread mood trackers seem limited in several, especially for an audience dealing with mental illness. ${ }^{1}$ These limitations may be linked to the lack of user experience research, as well as GUI (graphical user interface) research in interactive applications in the medical field.

In this paper we begin by discussing what mood trackers are used for. Subsequently we define the analysis criteria, and present the analysis of various examples, drawing conclusions about the potential role of Interaction Design in improving this type of product, and finally we make suggestions for future studies regarding the topics in question.

${ }^{1}$ Mental illness refers to "more than 400 diagnosable mental disorders (...) that disrupt a person's psychological functioning, and are characterized by alterations in thoughts, emotions and behaviors" [6]. 
Mood tracking is the act of registering through a period of time the mood one feels and how day to day life can affect mood; many times to try to find patterns within routines that can be either helpful or harmful towards overall wellbeing and mental health. This act of tracking one's mood has shown to be helpful when it comes to health management $[3,7]$. There are several tracking mobile applications in the market, from focusing on tracking the amount of water the user drinks per day, to how many cigarettes they smoke. These habit-tracking apps seem to always be connected to the users wanting/needing help to accomplish a positive change in their life. Mood trackers have some variances as well, from merely allowing the user to input their current mood, to associating the current mood with an event, people, or daily habits, such as exercising.

Mood trackers can be significantly improved by an Interaction Design intervention. For example, a user experience study and analysis of what users need and expect from mood trackers can provide designers with valuable insight that will allow them to better tend to the users' needs. Furthermore, involving therapists in the process of creating mood trackers can also provide a better understanding of what their clients' needs are.

This study analyses several of the most popular mood trackers currently in mobile applications stores. The criteria for choosing these mood trackers were both the order that they appeared when conducting a search in both Google Play Store and Apple Store, and their ratings according to users. This paper reports a first introductory analysis using some of the criteria we found important to implement in a future co-designed application with a therapist.

\section{$3 \quad$ Interaction Design within Mood Trackers}

From an interaction design perspective, mood tracker applications vary greatly from applications like T2 Mood Tracker - whose interface is somewhat outdated and narrow, allowing exclusively one thing: tracking moods (only the several moods it has predefined, never just one or two), and it does not do it very well from an user experience standpoint [8] -, to applications such as Youper - which tries to bring innovation into the mobile mental health world through artificial intelligence, in a chat format, to possibly try and establish rapport with the user.

One big contribution Interaction Design can make towards a better designed mood tracker starts with research. Cooper [9] begins his 
interaction design process with research, by comparing types of research (quantitative versus qualitative), and introduces the reader to "goal-directed design." Another good contribution interaction design can have is the fact that most mood trackers are made for mobile graphic interfaces, which can be a very helpful medium to carry daily use interfaces, however designing something for a smartphone screen is very different from designing a web browser or a computer application.

\subsection{Interaction design approach to the analysis}

The approach we took rests on the criteria defined by several interaction design authors $[11,12,13]$. Our research focused on authors who had previously defined rules for graphic user interfaces and mobile applications. These criteria are adapted to mobile.

The first criteria we chose to analyse was the target audience/stakeholder for each application, since previous studies have shown that some applications in the market are aimed at a general public and not people undergoing therapy $[3,10]$. However the constant change in the market compelled us to maintain this criteria.

The second one, was the input of emotions, focusing on whether it would allow for only one emotion or multiple emotions at the same time. Humans are complex beings, and it is often very difficult to translate all that we are feeling into one word or emotion. Furthermore, mood can consist of several emotions [4].

In terms of interaction design, we analysed the interaction types [11], which could be classified as instructing, conversing, manipulating, exploring, or responding. Instructing is defined as the user giving input to a system, which can range from typing in commands into a command line to speaking commands aloud. Secondly, conversing proposes a dialog between user and system. Manipulating requires the users to apply their knowledge of the world around them to the interface in question, either virtual or physical. Lastly, responding is defined as the system starting the conversation with the user and the user being able to choose whether or not to respond to it.

We further analysed interaction styles. Shneiderman [12] separates these interaction styles into five main categories: direct manipulation, menu selection, form fill-in, command language, and natural language. Direct manipulation is defined as simplifying the user's tasks in a familiar way or concept so that it is easier to understand for the user. The author provides examples such as the desktop metaphor and drawing tools in software. Navigation and menu selection, as the name suggests, focus on the use of menus for navigating the software. This interaction style provides users with an easily accomplished task "with little learning or memorization in just a few actions" [12]. Form fill-in is a common type of data entry, commonly used in registration forms for fields such as date of birth and country, it is usually 
pre-filled and requires only simple selection from the user. The previous three interaction styles were the only ones observed in the mood tracker applications. Command language requires a more frequent user and provides users with a strong feeling of being in control (e.g., programming) and natural language, although used in a lot of mobile phones nowadays, has not really been transferred over to a lot of mobile applications. Natural language can be anything from text-to-speech, to the user being allowed to give commands just by speaking (e.g., Siri, Alexa).

We then proceeded to analyse visual complexity of each individual application. For this, Miniukovich \& Angeli [13] provided some useful guidelines, mostly about how information can be displayed in mobile screens (Table 1). They separated visual complexity analyses of mobile applications into three different sections: amount of information, organization of information, and discriminability of information.

Table 1. The classification of visual complexity determinants. Adapted from Miniukovich \& Angeli [13].

\section{Information}

\begin{tabular}{|c|c|c|}
\hline Amount & Organization & $\begin{array}{l}\text { Discriminability } \\
\text { (Hierarchy) }\end{array}$ \\
\hline $\begin{array}{ll}\text { - } & \text { Clutter } \\
\text { - } & \text { Dominant colors } \\
\text { - } & \text { Color Depth }\end{array}$ & $\begin{array}{ll}\text { - } & \text { Symmetry } \\
\text { - } & \text { Ease of grouping } \\
& \text { prototypicality } \\
\text { - } & \text { Grid }\end{array}$ & $\begin{array}{ll}\text { - } & \text { Figure-ground } \\
\text { contrast } \\
\text { - } & \text { Edge congestion }\end{array}$ \\
\hline
\end{tabular}

The amount of information was defined because, from a psychological point of view, less clutter and information shown in a screen means less workload. Therefore, it is more usable in terms of interaction with the system. Adapted to the visual domain this translates into color dominance and color depth as the use of "too many dominant colors make the user perceive a GUI as complex" [13]. Lastly, clutter and amount of information presented to the user are also to be taken into consideration.

Organization of information can be explained by research in psychology that found a preference for symmetry and grid-based visual arrangements, as "regularity and repetition contribute to figural goodness" [13].

Discriminability of information can be defined as making the task of assimilating provided information easier for users. In small mobile screens being edge congestion, as the lack of open spaces makes it more difficult for the human eye to perceive information and figure-ground contrast, which if 
done well reduces the effort and speed with which users can assimilate the information provided.

An important observation we made was whether the applications used the aid of images in their interface, specifically in the tracking of moods, to understand each emotion.

We also analysed the applications' design in terms of occupying any space within therapy sessions and if their development was aided by specialists in the area.

\section{$4 \quad$ A First Exploratory Analysis of Mood Tracker Applications}

For this analysis, we chose 29 applications that were advertised in their respective application stores as mood trackers. However, upon using the applications, we excluded three for either paid content or because they were not actually mood trackers, but mental health related "testing" applications. We were left with 26 applications ${ }^{2}$ that were analysed. Out of the 26 analysed applications, 11 were iOS-only applications, seven were Android OS only, and four were available for both operating systems.

The first observed criteria was stakeholders (target audience), and, as Caldeira [3] predicted, most of the analysed applications are aimed towards a more general public: correspondingly, from the 26 reviewed applications, 19 were aimed at a general public.

The second criteria concerned emotion input, that is, if more than one emotion was possible to be input into each mood tracker. We found that from the 26 analysed applications, only eight allowed for multiple emotion input, and two of these allowed for input of multiple emotions exclusively, which were predefined by the application itself.

Subsequently, we proceeded with the Interaction Design analysis. Regarding interaction types [12], all applications used touch, since they were designed for smartphones. Nine also made good use of sliders, which fall under the "instructing" category concerning interaction types. However, there are several layers that can be explored and several applications (15 out of the 26) had a reminder option, in which the user tells the application that he wants to be reminded to interact with it, which can fall under the "responding" category. If the users choose to respond, it then turns into "instructing". One of the applications in particular used an AI (artificial intelligence) based chat system, in which the AI would ask questions and give answers as options. This can fall under all 3 categories: instructing, conversing, and responding.

2 The full list of analysed applications is as follows: T2 Mood Tracker, Moodnotes, Daylio, Moodpath, Youper, Mood Log, Mood Tracker, Breeze, Moody, Emoly, iMood, Modeline, MoodPanda, Feelic, Mood App, Moodflow, Jade, Free Mood, Tracker, Puncher, Mood Patterns, Moodily, Pixels. 
From Shneiderman's [12] approach to interaction styles mentioned in the previous section, smartphone applications seem to mostly use navigation and menu selection. Some of the analysed applications used this style exclusively, while 10 of them also required direct manipulation, mostly seen in the ones that made use of sliders.

We found that image use is very popular among mood tracker applications, especially the use of emoji/face icons associated with a word. However, from the 18 applications that used images on their interface, stepped away from the traditional association of an emoji/face with a corresponding emotion word; images were used solely with the purpose of helping put an interpretation to the words.

Dividing visual complex into the three classifications established by Miniukovich \& Angeli [13] (Table 1), we started by analysing the "amount" column, finding that only 10 out of the 26 applications analysed showed a clear dominant color and had no clutter, meaning that the spacing in between words and images allowed for a clear and easy understanding of the application. A few applications (3) seemed to follow no rules and present no contrast or depth, making them hard to use and straining the user's eye, also having too many colors or too much white and a lot of blank space.

Regarding organization of information, a significant amount of the analysed applications (15 out of 26) made use of a layout grid, a basic structure as the base of the $\mathrm{UI}^{3}$ (user interface) design. The usage of grid has several advantages in terms of visual interface design, from a usability standpoint, it helps regularise the position of elements. Having a standard layout grid can also make the designing and using of the application easier, as every page follows the same layout, it is more aesthetically pleasing, and reading and understanding the layout is also easier from the user's perspective $[9,13]$.

The two topics mentioned in discriminability of information are contrast and edge congestion, and most visual interfaces nowadays tend to have a good contrast. According to our findings, 14 out of the 26 applications had a good use of the figure-ground contrast. Text was clear and easy to read and there were clear distinctions between figure/text and background. However, a considerable amount of the applications analysed suffered from edge congestion, that is, a lack of open space, the need to share too much information in small screens often leads to this common problem [13]. Seven out of the 26 applications presented some form of this problem. Conversely, however, there were some applications (3) that had too much free space, as if the screen was too big for the information it was carrying, causing them to have a lot of blank, usually white, spaces.

Following this, we aimed to find whether these applications took into account people currently undergoing therapy, and whether there was a space service" [15].

${ }^{3}$ User Interface is the "interface between users and a product or 
they could occupy in the therapy space to help patients. Also taken into account was whether mental health specialists or doctors were involved in the creation of each application. When it comes to space occupied in therapy, which is somewhat related to the stakeholders, 19 out of the 26 applications had no mention or applicable use for therapy, while six of them had a possibility to create a report. This report could be shared with a doctor. Some applications did it better, providing a PDF version of it. Others required the doctor to sign up to the application to be able to view it. Only one of the 26 applications was recommended by therapists and was advertised as a companion for therapy. Finally, only three of the analysed applications were aided by therapists or based on cognitive-behavioural therapy. This indicates that, possibly, most of the applications people are using do not even have scientific methods or approaches, which means that in some cases, they can be more harmful than good, especially if the patient is suffering from mental health issues and trying to get better without recurring to therapy or any type of doctor.

\section{$5 \quad$ Limitations}

The biggest limitation of this study is that the selected criteria for choosing the applications had to exclude the paid features and the paid apps available in the mobile apps stores. Only the two biggest application stores were considered: Google Play Store and Apple Store; and even though these may be the ones with most applications, there can be ones that are not yet in these platforms that we missed because of the defined criteria.

The constant update and renaming of mood tracker applications was a limitation during this study. Because the study was not carried out during a small time frame, a lot of the applications first studied were either deleted, renamed, or completely redesigned, or both, and this factor made the whole process more difficult and did not allow us to analyse as many applications as initially intended.

Finally, the study presents itself as a first exploratory attempt into the mood tracker ecosystem that has developed in recent years. More studies with the intention of deepening the collected knowledge will be conducted in the context of this research.

\section{Conclusions}

Reviewing this first approach at how interaction design could improve mood trackers as they currently are, firstly a lot more user-centered design can be used. Defining a target audience seems to be lacking when it comes to the designing of mood trackers, and with no target, applications just end up falling 
short of what they could achieve and having no concrete goals or objectives. Specific users have specific needs, and targeting applications to the general public can exclude these users that might really benefit from having their needs met.

Secondly, what appears to us as a worrying conclusion of this study is that most of these applications have no support from mental health specialists. Considering that, most likely, people who look up these applications may be considering the state of their mental health, having an application that is not approved by a specialist can be more damaging than good to the mental health of users.

An interaction design and co-design approach to these applications, with good user research supporting it and taking into account what the users expect and need from these kinds of applications, seems to be a better solution than what we currently find. Knowing the target audience's expectations and having the support of mental health specialists can help designers turn a simple habit tracking application into something that can be used as support for therapy.

Furthermore, applications, specially medical-related ones, should have basic guidelines to follow, so that the market is not flooded with several applications that all serve the same purpose or are not credible to do what they aim to. From an interaction design standpoint it would even be useful for the designers to have these guidelines, on what can or cannot be damaging to a user seeking this type of application.

Applying different interaction styles and types aims to make the interaction with the application more dynamic in a way that supports the user and does not condition the answers. In addition, trying to establish a connection with the user through the design of the application and facilitating the use of it, making the possible daily use of this application something to look forward to is the aim.

The current state of eHealth and mHealth needs guidelines, both in terms of health and design. A co-design between mental health specialists and interaction designers with dedicated usability and $\mathrm{UX}^{4}$ (user experience) research can be a good first step in the definition of these guidelines. Furthermore, mood track applications never seem to get out of the comfort zone of using words only to describe emotions, or simple "emojis" that are associated with these words. This makes us wonder whether having the user find a deeper connotative meaning in different images can help an in-between therapy reflection.

A space for reflection is very important, and although some of these applications do provide notes associated with the moods, a journaling space of different sorts could improve the reflection time, and having all of those points gathered in a single app. Adapting other types of interaction that go

4 "User Experience is simply how people feel when they use a product or service". [14] 
beyond touch, such as voice or using, like previously mentioned, images or videos, as another medium besides words could be a better approach to fully take advantage of a mental health guide that, through a mobile application, is with the patient at all times.

For future studies, different types of interactions should be applied, but also, different approaches to what mood trackers are and what they can become, for example, in the context of therapy, or just as a support system for the users. The next step of the current research is re-designing the concept of mood trackers and adapt them to patients who are currently undergoing therapy, to make these mood trackers helpful both for the patient in between therapy sessions and for patient and therapist during the sessions, and to provide a better opening and discussion topics to jumpstart the sessions.

\section{References}

1. Chan, S., Torous, J., Hinton, L., \& Yellowlees, P. (2015). Towards a framework for evaluating mobile mental health apps. Telemedicine and E-Health, 21(12), 1038-1041.

2. East, M.L. \& Havard, B.C. (2015). Mental health mobile apps: From infusion to diffusion in the mental health social system. JMIR Mental Health, 2(1), 1-14.

3. Caldeira, C., Chen, Y., Chan, L., Pham, V., Chen, Y., \& Zheng, K. (2017). Mobile apps for mood tracking: an analysis of features and user reviews. Retrieved from https://www.ncbi.nlm.nih.gov/pmc/articles/PMC5977660/

4. Desmet, P.M.A. (2015). Design for mood: Twenty activity-based opportunities to design for mood regulation. International Journal of Design, 9(2), 1-19.

5. Bush, N.E., Ouellette, G., \& Kinn, J. (2014). Utility of the T2 mood tracker mobile application among army warrior transition unit service members. Military Medicine, 179(12), 1453-1457.

6. Thieme, A., Wallace, J., Meyer, T.D., \& Olivier, P. (2015). Designing for mental wellbeing: towards a more holistic approach in the treatment and prevention of mental illness. In Proceedings of the 2015 British HCI Conference - British HCI '15 (p. 1-10). New York: Association for Computing Machinery.

7. Nicholas, J., Larsen, M. E., Proudfoot, J., \& Christensen, H. (2015). Mobile apps for bipolar disorder: A systematic review of features and content quality. Journal of Medical Internet Research, 17(8), e198.

8. Saperstein, A. (2014). Comparison of two PTSD apps: PTSD Coach and T2 Mood Tracker. Retrieved from https://onemindpsyberguide.org/apps/t2-mood-tracker/

9. Cooper, A., Reimann, R., \& Cronin, D. (2007). About Face 3: The Essentials of Interaction Design (3rd ed.). Indianapolis, IN: Wiley Publishing.

10. Stawarz, K., Preist, C., Tallon, D., Wiles, N., \& Coyle, D. (2018). User experience of cognitive behavioral therapy apps for depression: An analysis of app functionality and user reviews. Journal of Medical Internet Research, 20(6), 1-16.

11. Sharp, H., Rogers, Y., Preece, J. (2019). Interaction Design: beyond human-computer interaction (5th ed.). John Wiley \& Sons. 
12. Shneiderman, B., Plaisant, C., Cohen, M., Jacobs, S., Elmqvist, N., \& Diakoppoulos, N. (2018). Designing the User Interface: Strategies for Effective Human-Computer Interaction, 6th edition. In Society (6th Edition). Essex, England: Pearson Education.

13. Miniukovich, A. \& Angeli, A. (2014). Visual impressions of mobile app interfaces. In NordiCHI '14: Proceedings of the 8th Nordic Conference on Human-Computer Interaction: Fun, Fast, Foundational (p. 31-20). New York, NY: Association for Computing Machinery.

14. Soegaard, M. \& Dam, R.F. (2018). The Basics of User Experience Design. The Basics of User Experience Design, 73. Retrieved from interaction-design.org 